\title{
EDITORIAL from Jorge Mateu
}

\author{
Jorge MATEU
}

I would like to introduce myself as the new Editor-in-Chief for the term 2022-2024 of the Journal of Agricultural, Biological, and Environmental Statistics (JABES) and welcome my team of associate editors. I am delighted to work for this lively journal with an exciting present and future impacting on the statistical and subject matter fields.

Briefly about me I graduated in Mathematical Sciences at University of Valencia (Spain) and fulfilled the $\mathrm{PhD}$ at the University of Valencia with long visiting periods to University of Lancaster (UK), with Prof. Peter Diggle. I am currently a full professor of Statistics with the Department of Mathematics at University Jaume I of Castellon, where I have worked for the past 25 years. My main fields of interest are stochastic processes in their wide sense with a particular focus on spatial and spatiotemporal point processes, but also on geostatistics and areal spatial data. My research lies at the intersection of statistics, computational sciences and natural and social sciences with a wide focus on Data Science. Large projects in crime and environmental data analysis, where combination of statistical methods and machine learning methods are at the core of the approach, and in public health and epidemiology are currently taking most of the time of my research group. Thus, part of my research is methodological and the other part involves the direct application to real data; as a consequence, my publications are divided between methodological statistical journals and more applied ones. Statistical science has been at the core of the large number of organized workshops and corresponding special issues. And I will try to bring my experience to further foster JABES at introducing novel statistical methods to solve practical problems in the agricultural, biological and environmental sciences.

A bit of history The Biometric Society was originally founded in September 1947, with a clear mission to promote the development and application of statistical and mathematical theory and methods in the biosciences, including agriculture, biomedical science and public health, ecology, environmental sciences, and forestry, among other disciplines. Later renamed as International Biometric Society, IBS (http://www.biometricsociety.org/) is a worldwide society with members from over 70 different countries, and structured in rec-

J. Mateu ( $\varangle$ ) Department of Mathematics, University Jaume I of Castellon, Castellón de la Plana, Spain (E-mail:mateu@uji.es).

(C) 2022 International Biometric Society

Journal of Agricultural, Biological, and Environmental Statistics, Volume 27, Number 1, Pages 1-3 https://doi.org/10.1007/s13253-022-00489-z 
ognized Regions, over half of them representing members from developing countries. IBS promotes and fosters two leading scientific journals: Biometrics and JABES, which are the outward face of the Society.

Biometrics, a top scholarly publication reporting work promoting the use of statistical and mathematical theory and methods in the biosciences, was founded in 1945 as Biometrics Bulletin, and later renamed as Biometrics in 1947. In the early 1990s, a strong feeling within IBS raised supporting arguments in favor of a new journal with papers structured around a motivating example, keeping a balance across the agricultural, biological and environmental sciences. Discussions between IBS and the American Statistical Association (ASA) (http://www.amstat.org/) resulted in the launch in 1996 of JABES, jointly published by the two societies. Since then, JABES has been successful at maintaining such a balance, with input from authors with a large geographic spread, and publishing papers that introduce new statistical methods to solve problems in the agricultural, biological and environmental sciences.

My vision Statistics, the science of obtaining useful information from data, taking its variability and uncertainty into account is a cornerstone of science, health, economics, medicine and more, and benefits society as a whole. At JABES, we aim to recognize contributions with a significant impact and relevance to Society, underlying the important role and intellectual content of statistics. In this sense, statistical work can be found under a variety of flags, such as big data, biometrics, classification, data analysis, data collection, data mining, data science, data visualization, design of experiments, environmetrics, genomic statistics, machine learning, multivariate analysis, pattern recognition, statistical computing, and statistical learning.

JABES should be home for Data Science broadly defined as the science of learning from data, incorporating advances in computation and data analytics, with statistical theory and inference for problems coming from the branches that sustain the journal. We are increasingly handling large and rich data that pose a number of challenges in fully realizing the potential of these data, including, for example, understanding quality and sources of bias in data derived from multiple sources, and handling the complexity and high-dimensionality of large, longitudinal and high velocity data sources. There is a need for evidence on how (and in which settings) data science methods add value to traditional statistical approaches and can be used translate data into tools that can benefit society.

I would like to encourage interdisciplinary submissions that involve collaboration between statisticians and other data scientists to find solutions to these challenges, through innovative methodological developments and applications, bringing together data science and statistics. The innovative methodology should be directly motivated by real-world data problems in agricultural, biological and environmental settings. For example, motivating examples should include studies aiming to use data science which might involve data engineering, computational methods (e.g., pattern recognition, machine learning, artificial intelligence), or data visualization.

A way to put this into practice for the benefit of the journal is to plan and organize a number of topical special issues on timely research problems. One of my priorities as editor is to seek quality papers and allow JABES to distinguish itself. I use the word quality in 
terms of the impact on the statistical and subject matter fields. I seek innovation in statistical methods that help advance our field, but very much motivated by real problems. It is a priority to publish the most appropriate statistical methods to address important problems in the agricultural, biological, and environmental sciences.

I very much welcome my team of 29 associate editors, representing USA, Europe, Australia, New Zealand, China, Canada, and Saudi Arabia. I look forward a continued interaction with all of them over the coming years. And last, but not least, I would like to thank the preceding EiC, Brian Reich, who has made all efforts to make the transmission over smooth. Brian has brought JABES at the end of his term to excellent figures in terms of high position at several official rankings, number of high-quality papers, quick final decisions, number of article downloads and open access, and much more. We do hope to keep things in that line improving wherever possible.

\section{[Accepted January 2022. Published Online February 2022.]}

Publisher's Note Springer Nature remains neutral with regard to jurisdictional claims in published maps and institutional affiliations. 